Kaur Alttoa

\title{
EINIGE KORREKTUREN BEZÜGLICH DER KIRCHEN ST. JACOBI IN RIGA UND DER ZU LUGGENHUSEN (LÜGANUSE) IN WIERLAND (VIRUMAA)
}

In einem im Jahr 2013 erschienenen Aufsatz zur Kirche zu Urbs (Urvaste) habe ich auch ein wenig die Rigenser Jakobskirche behandelt. Unter anderem habe ich auf die Ecksäulchen des Chorraumes verwiesen, die Basen und Kapitelle mit Eckblättern besitzen. ${ }^{1}$ Obwohl diese mit Bronzefarbe sorgfältig übergemalt sind, können ihre Formen und Einzelheiten deutlich erkannt werden. Es scheint so, dass auf ihrer Grundlage ein wichtiger Eckpunkt für die Datierung der Kirche abgeleitet werden kann. Im Mai 2017 besuchte ich St. Jacobi in Riga zusammen mit dem Architekten Ilmars Dirvieiks. Er erklärte mir, dass alle jene Kapitelle und Basen aus Mörtel in Wirklichkeit am Ende des 19. Jahrhunderts im Verlauf von stattfindenden Restaurierungsarbeiten gegossen wurden.

Diese Information erreichte mich als wirkliche Überraschung. Das Thema der Kapitelle der Skulpturen der Jakobskirche brachte im Jahr 1960 Voldemar Vaga in den wissenschaftlichen Umlauf. Er publizierte 
nämlich die Fotos von drei Kapitellen und einem Sockel und datierte sie auf das Ende des 13. Jahrhunderts. ${ }^{2}$ Das Werk Vagas wurde ziemlich oft zitiert und zur selben Zeit auch kritisiert. Gleichzeitig habe ich auch keine Bemerkung angetroffen, dass mit den Kapitellen der Jakobskirche irgendetwas nicht stimme. Auf jeden Fall besaß ich keinen Grund daran zu zweifeln, dass dieses Steindekor aus dem Mittelalter stamme. Natürlich hätte ich mich in das 1892 erschienene Buch Wilhelm Neumanns vertiefen sollen, welches das mittelalterliche Riga behandelt. Dort gibt es auch ein umfangreiches Kapitel über die Jakobskirche ${ }^{3}$, doch vom Eckdienste und den Kapitellen des Chorraums schreibt Neumann kein Wort. Hinterher erscheint die Ursache als klar, damals existierten sie einfach noch nicht.

Die Entstehungsgeschichte der Kapitelle erhellt der Architekt Wilhelm Bockslaff, der in den Jahren 1895-1902 die Restaurierungsarbeiten der Jakobskirche geleitet hat. ${ }^{4}$ Denn in der Bibliothek der Universität Poznań (Posen) ${ }^{5}$ werden zwei Schreibmaschinenmanuskripte Wilhelm Bockslaffs aufbewahrt: „Meine Erinnerungen an die Wiederherstellung der Kronsund Ritterschafts-Kirche zu ST. JAKOB in Riga“" und „Aus der Arbeit eines baltendeutschen Architekten an den alten Kirchenbauten Rigas und Dorpats ". 7 Das letztere Manuskript trägt die Jahreszahl 1943, beim ersteren fehlt die Datierung.

Bockslaff schreibt: „Beim Ausschachten der Erde und Senkung des Fussbodens auf eine dem Kirchenschiffes angemessene Höhenlage kamen in allen vier Ecken des Chorquadrats die unteren Enden runder DreiviertelDienste mit romanischen Basen nebst Eckblättern zum Vorschein. Die Basen befanden sich in einer Tiefe von etwa $2 \mathrm{~m}$. unter dem bisherigen Fussboden und etwa einen halben Meter unter dem Horizont der Schwelle des wiederentdeckten Nordportals des Schiffes. Diese Reste verblieben

2 Voldemar Vaga, Problema prostranstvennoj formy v srednevekovoj architekture Latvii i Estonii. Tartu Riikliku Ülikooli Toimetised, 86 (Tartu: Tartu Riiklik Ülikool, 1960), 11-13. Die deutschsprachige Ausgabe des Buchs: Voldemar Vaga, Das Problem der Raumform in der mittelalterlichen Baukunst Lettlands und Estlands (Tartu: Staatliche Universität Tartu, 1960), ist ohne Illustrationen. Entsprechender abschnitt: ibidem, 16

3 Wilhelm Neumann, Das mittelalterliche Riga. Ein Beitrag zur Geschichte der norddeutschen Baukunst (Berlin: Julius Springer, 1892), 24-27.

4 Die bis jetzt umfangreichste Übersicht über den Architekten: Gunārs Priede, Arhitekts Vilhelm Bokslafs un Rīga. Der Architekt Wilhelm Bockslaff und Riga (Riga: Jāṇa Sēta, 1997).

5 W. Bockslaff siedelte sich im Verlauf der Umsiedlung im Jahr 1939 in Posen an, wo er 1945 verstarb

6 Tatsächlich ist ein Teil dieses Manuskripts auch der Renovierung der Johanniskirche in Dorpat (Tartu) gewidmet.

7 Biblioteka Uniwersytetu im. A. Mickiewicza w Poznaniu. Zbiory specjalne, Nr. 2046, 41-61. unberührt an ihrer Stelle und über dem neuen Fussboden wurden nach dem Muster dieses Fundes neue Eckdienste in Putz-Technik nebst nach den vorgefundenen modellierten Basen und Frühgotischen Kapitälen in Cementguss hergestellt. ${ }^{\prime 8}$ Und an anderer Stelle: „Der einzige Fund von Bedeutung war die Entdeckung der unteren Teile rundlicher Ecksäulchen aus Kalkstein mit romanischen Eckblatt-Basen im Chorquadrat und über dem Fussboden konnte festgestellt werden, dass diese Eckdienste in ihren oberen Teile abgehauen worden waren. Da das bestehende alte auffallend hochgespannte Domical-Gewölbe dieses Raumes ohne Rippen auf den scharfen Grad gemauert war, hat beim Aufmauern des Chorbaus wohl die Absicht bestanden, wie im Dom, ein Rippengewölbe anzuwenden. Die fehlenden oberen Teile liess ich über dem neuen Fussboden bis zum Gewölbefuss in Cementguss neu herstellen und versah sie mit Capitälen nach dem Vorbild derjenigen im Domkreuzgang. Die gefundenen Reste verblieben unter dem Fussboden." ${ }^{\prime 9}$

Damit erscheint es als klar: Im Chorraum der Jakobskirche sind Säulenbasen mit Eckblättern gewesen, doch die heute sichtbaren Eckdienste sind mit Sicherheit neuzeitlich.

$* * *$

In einem kurzen Beitrag habe ich die Vermutung vorgestellt, wie die Gewölbe der Kirche zu Luggenhusen in Wierland komponiert gewesen sein könnten. ${ }^{10}$ Bekannterweise war die Parochialkirche zu Luggenhusen anfangs ein einfacher Kastenbau ohne Gewölbe gewesen. Erst im 15. Jahrhundert errichteten Meister aus Reval (Tallinn) in der Kirche ein Gewölbe, wobei das Langhaus zweischiffig gestaltet wurde. Heutzutage wird dies in der westlichen Wand des Langhauses durch erhalten gebliebene Reste eines Gurtbogens markiert. Im Jahr 2013 wurden auch die Überbleibsel von Pfeilern ausgegraben. ${ }^{11}$ Wie aber wurde der östliche Bereich des Langhauses gestaltet war, also vor dem Altar - darüber fehlten jegliche Angaben. Ich bot die folgende Vermutung an, dass das

\footnotetext{
8 Biblioteka Uniwersytetu im. A. Mickiewicza w Poznaniu. Zbiory specjalne, 42.

9 Ibidem, 50

10 Kaur Alttoa, „Die Gewölbekomposition der Kirche in Luggenhusen/Lüganuse“, Baltic Journal of Art History, 9 (2015), 225-237.

11 Villu Kadakas, Tõnno Jonuks, ,Archaeological Studies in the Church and Churchyard of Lüganuse“, Archaeological Fieldwork in Estonia 2013 (Tallinn: Muinsuskaitseamet, 2014), 143.
} 


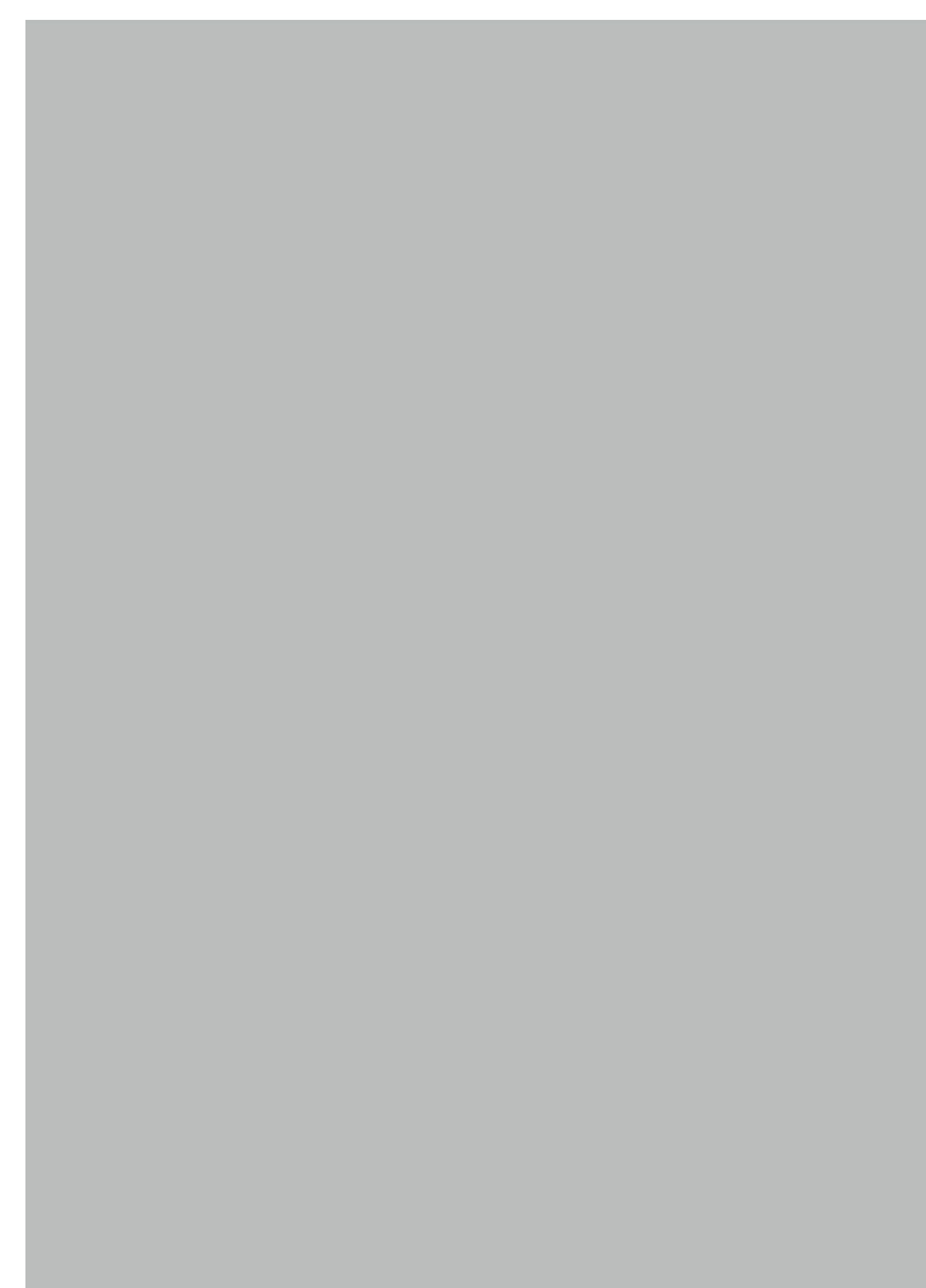

Abb. 1. Innenansicht der Kirche zu Luggenhusen am Ende des 19. Jahrhunderts. Foto aus der Sammlung von Reinhold Guleke.

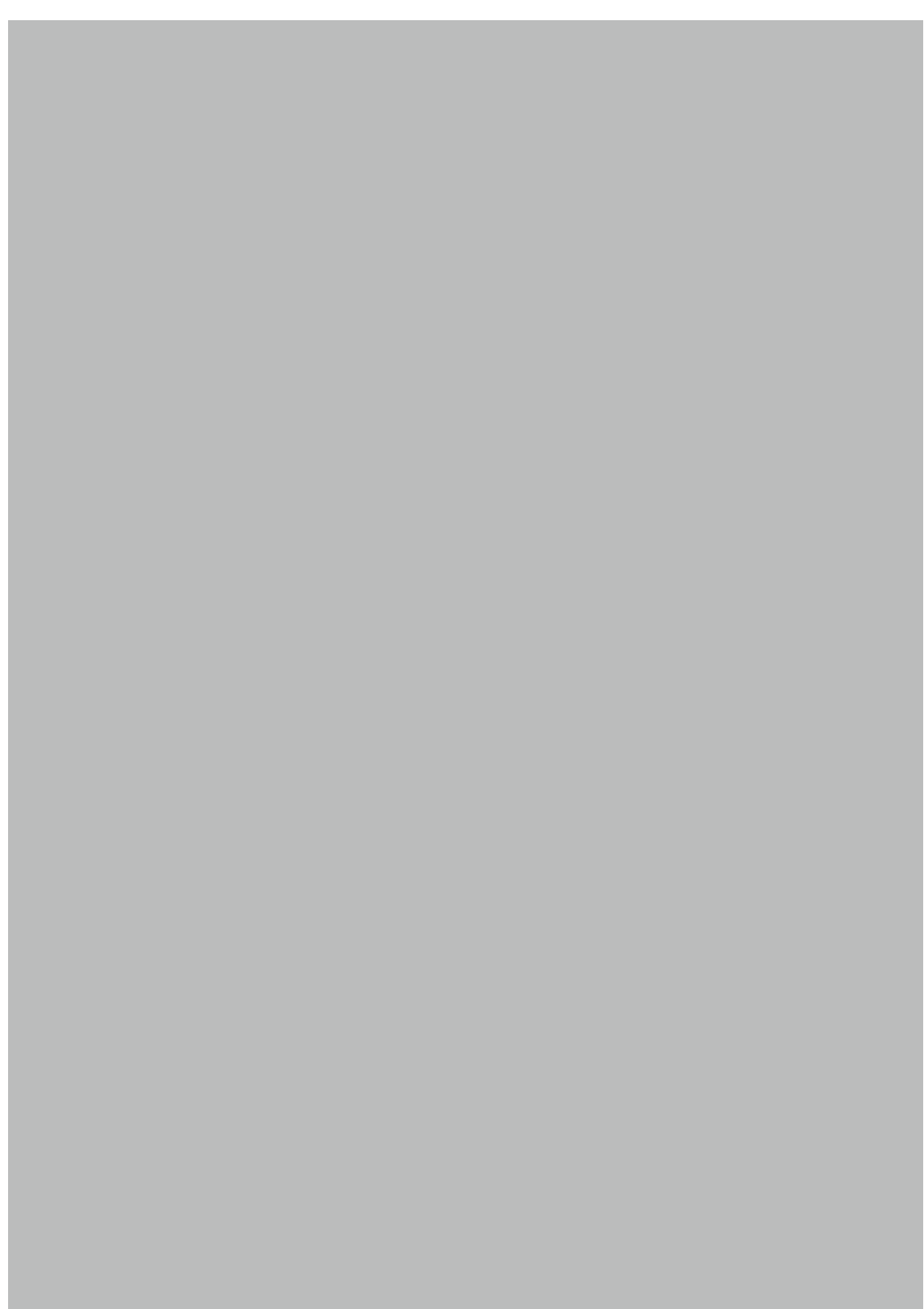

Abb. 2. Östliche Wand des Langhauses der Kirche zu Luggenhusen am Ende des 19 Jahrhunderts. Foto aus der Sammlung von Reinhold Guleke. 
Gewölbe in Luggenhusen so gestaltet gewesen sein könnte wie in einer anderen nordestnischen Kirche - eben wie in Kegel (Keila). Dort gehen vom östlichen Pfeiler diagonal zwei Gurtbogen aus. Auf diese Weise bildet sich vor dem Altarraum ein zentraler Bereich, der auf diese Art für den von den Pfeilern in zwei Stücke zerteilten Raum kompensiert. Natürlich handelte es sich um eine Hypothese.

Im Mai 2017 beschäftigte ich mich mit dem Archiv des Architekten Reinhold Guleke, das sich in der Lettischen Nationalbibliothek (Latvijas Nacionālā bibliotēka) befindet. ${ }^{12}$ Unter anderem finden sich dort auch Fotos des Interieurs der Kirche zu Luggenhusen zur Zeit der Jahrhundertwende auf das 20. Jahrhundert. ${ }^{13}$ Auf diesen Bildern ist zu erkennen, dass damals Fragmente der Gurtbogen sowohl an der Längswand als auch, dies ist aber wichtiger, an der Ostwand des Langhauses erhalten waren. Denn dort befanden sich über dem Triumphbogen ebenfalls Reste des Gurtbogens. ${ }^{14}$ Damit war der östliche Teil des Langhauses ähnlich gestaltet wie andere Joche der Kirche und die Ähnlichkeit mit der Gewölbekomposition in Kegel ist unrichtig. Ein Parallelbeispiel für die Gewölbe in Luggenhusen ist stattdessen die Kirche zu Kreuz (Risti) in Harrien (Harjumaa).

Villem Raam hat einmal gesagt: „Wenn wir darüber nachdenken, wie etwas vor 700 Jahren erschaffen wurde, dann irren wir uns dabei ausgesprochen viel. Und ich würde sagen, unsere Arbeit - ich denke an meine Arbeit und ebenfalls an Deine Arbeit - besteht in einem großen Umfang darin, die ganze Zeit die eigenen Fehler zu korrigieren. Wir machen Fehler und stellen falsche Hypothesen auf, wir korrigieren sie und darin bestehen eben unsere Entwicklung, unser Unglück und unser Glück. ${ }^{\prime \prime}$ Dieser knappe Beitrag zählt zur Kategorie der Verbesserungen von Fehlern.
Kaur Alttoa: Some Corrections Regarding the St Jacob’s Church in Riga and LÜganuse Church in Virumaa

Keywords: St Jacob’s Church in Riga; St Jacob’s Church in LÜGANUSE

\section{CV}

Kaur Alttoa (b. 1947) has been a long-time scholar and teacher of art history at the University of Tartu and he is one of the foremost specialists of the history of medieval architecture in the Baltic region. His objects of interest have included both medieval fortresses and sacral buildings. Among his major contributions stands out the restoration and study of the St John's Church in Tartu, which was also published as a monograph in 2011.
12 Lettonica und Baltica Abteilung der Lettischen Nationalbibliothek. Archiv von Otto Bongs.

13 Ibidem, 2512 (Luggenhusen).

14 In den Jahren 1901-1902 erfolgten in der Kirche Renovierungsarbeiten. (Eesti Evangeelium Luteriusu Kirikud, hrsg. von Bruno Ederma, Asta Jaik (Tartu: K. Jaik, 1939), 91.) Offensichtlich wurden damals auch die Reste des Gurtbogens herausgehauen.

15 „Villem Raam restaureerimisest ja muust (Villem Raam über Restaurierung und über anderes)“, Villem Raam. Bibliograafia, hrsg. von Kaur Alttoa, Eve Palginõmm (Tallinn: Eesti Muinsuskaitse Selts, 
